\title{
Effectiveness and satisfaction evaluation of patients submitted to TMJ arthrocenthesis: a case series
}

\section{Mariana Vasconcelos da Cruz GOUVEIA \\ Jimmy Charles Melo BARBALHO Edwaldo Dourado PEREIRA JÚNIOR Mirella Marques das Mercês NASCIMENTO \\ Belmiro Cavalcanti do Egito VASCONCELOS}

Universidade de Pernambuco - UPE, Dental School, Department of Oral and Maxillofacial, Camaragibe, PE, Brazil.
Declaration of Interests: The authors certify that they have no commercial or associative interest that represents a conflict of interest in connection with the manuscript.

Corresponding Author:

Jimmy Charles Melo Barbalho

E-mail: jimmybarbalho@hotmail.com

DOI: 10.1590/1807-3107BOR-2015.vol29.0050

Submitted: Jul 28, 2014

Accepted for publication: Jan 08, 2015

Last revision: Feb 10, 2015

\begin{abstract}
The aim of this study was to evaluate the effectiveness and level of satisfaction of patients who underwent TMJ arthrocentesis under local anesthesia by considering the following parameters: joint pain, mouth opening, mastication, and satisfaction. Fourteen patients (13 women and one man; mean age, 37.6 years with TMJ arthralgia were selected. The patients underwent arthrocentesis under local anesthesia, and pain intensity was measured before, during, and 1 year after treatment using a visual analog pain scale (VAS 0-100 mm). A Likert scale was used to assess patients' opinion regarding the improvement of mouth opening and mastication. After treatment, patients were questioned on levels of satisfaction provided by arthrocentesis in regard to their quality of life. At the end of 1 year, patients showed a marked improvement in their pain clinical picture $(p<0.0001)$. Mouth opening and mastication, evaluated separately and then correlated $(\mathrm{R} 2=0.925$, $\mathrm{p}<0.0001)$, also showed a marked improvement. When questioned, all patients reported being satisfied with the treatment outcome.
\end{abstract}

Keywords: Temporomandibular Joint Disorders; Temporomandibular Joint; Arthralgia; Patient Satisfaction.

\section{Introduction}

Temporomandibular disorders (TMD) are conditions that can affect the temporomandibular joint (TMJ), masticatory muscles, and associated structures and can clinically manifest as craniofacial pain, limitation of mouth opening, and joint noises. ${ }^{1,2}$ Over $30 \%$ of the population suffers from TMD and have their quality of life severely affected by this condition. ${ }^{2}$ TMD can be treated by either conservative or surgical treatment. Conservative treatments include occlusal splints, physical therapy, thermotherapy, administration of myorelaxant drugs, analgesics, and anti-inflammatory drugs as well as laser therapy. Surgical treatments can be invasive (open TMJ surgery) or minimally invasive, including arthrocentesis and arthroscopy. ${ }^{3}$ Arthrocentesis intervention is most commonly used in patients not responding to conservative treatment. The lysis mechanical action and joint space washing break adhesions within the joint and remove inflammatory mediators, such as interleukins and cytotoxins, which are responsible for chronic pain; thus, pain relief allows the improvement of mouth opening and mastication. ${ }^{4}$ Arthrocentesis is the least invasive TMJ surgery, offers low risk, and can be performed on an outpatient basis under 
local anesthesia or conscious sedation. ${ }^{5}$ The aim of this study was to evaluate the effectiveness and the level of satisfaction of patients who underwent TMJ arthrocentesis under local anesthesia.

\section{Methodology Study design}

This prospective study was performed at the Universidade de Pernambuco - UPE between March 2012 and March 2013. The study was approved by the Research Ethics Committee (CEP/UPE) and registered under number 04474012.4.0000.5207. All patients signed an informed consent agreement. Patients were recruited at the Department of Oral and Maxillofacial Surgery of UPE. The inclusion criteria were a minimum age of 16 years, anterior disc replacement with or without reduction, mouth opening limitation of articular origin, and pain of articular origin, and the exclusion criteria were pain of muscular origin, systemic rheumatic disease, pregnancy, TMJ ankylosis, previous TMJ surgery, current use of antiinflammatory drugs, corticosteroids, muscle relaxant or anti-depressants, and systemic disease.

\section{Subjects}

Fourteen enrolled patients, (13 females and one male; mean age, 37.6 years; SD 11.8) showing TMJ arthralgia (classified according to Research Diagnostic Criteria for Temporomandibular Disorders, RDC/TMD), underwent arthrocentesis as an initial treatment. Data was collected by a specific questionnaire in which the intensity of pain before, during, and after 1 year of the procedure, the patients' opinion regarding the improvement of mouth opening and mastication, and their satisfaction with the outcome of the procedure were determined.

\section{Arthrocentesis procedure}

The procedure started by blocking the auriculotemporal nerve followed by anesthesia of the posterior deep temporal and masseteric nerves. A straight line was drawn on the skin from the middle portion of the tragus of the ear to the lateral corner of the eye; two points for the insertion of needles were marked on this line: the first, posterior point was marked at a $10 \mathrm{~mm}$ distance from the tragus and $2 \mathrm{~mm}$ below the same line; the second point was marked at a $20 \mathrm{~mm}$ distance ahead of the tragus and $10 \mathrm{~mm}$ below the line. A sterile mouth opener was placed between the dental arches on the contralateral side of the arthrocentesis to enable downward and forward displacement of the mandible head; thus, allowing easier access to the posterior recess of the upper compartment of ATM. An 18 G needle was introduced at the posterior point (first marking), which was connected to a $5 \mathrm{ml}$ syringe, through which a $4 \mathrm{~mL} 0.9 \%$ saline was administered to distend the joint space. Another similar needle was introduced in the same distended compartment ahead of the first (second marking). Then, $300 \mathrm{ml}$ of $0.9 \%$ saline was injected by the first needle and collected by the second. No substances or drugs were added to the injected solution, and all procedures were performed by the same clinician. Patients were instructed to take $600 \mathrm{mg}$ Ibuprofen for 3 days in case of pain.

\section{Measurement variables}

The Visual Analog Scale (0-100 mm VAS), which helps to assess the subjective pain intensity of the patient (before, during, and 1 year after the procedure) was used. Mastication and mouth opening variables were assessed by the Likert scale, revealing the level of agreement or disagreement with the patients' opinions regarding these variables. This scale presented a series of five propositions, among which the respondent was asked to select one: strongly agree (score 5), agree (score 4), no opinion (score 3), disagree (score 2) and strongly disagree (score 1). The level of the patients' satisfaction with the procedure was obtained by a questionnaire with the response options "Yes" and "No".

\section{Statistical analyses}

Statistical analyses were performed with SPSS statistics version 17.0 software (SPSS Inc., Chicago, USA); pain intensity frequencies (before, during, and 1 year later) were determined and compared using the Chi-square test; means and standard deviations of pain intensity (before, during, and 1 year later) were determined and compared using Student's t test; The Kolmogorov-Smirnov test was used to verify the normal distribution of continuous variables. The Pearson correlation coefficient was obtained for 
comparisons between mouth opening and mastication. Differences were considered significant when $p<0.05$.

\section{Results}

Fourteen patients, 13 females (92.9\%) and one male, were included in the study. The patients' ages ranged between 22 and 57 years, with an average of 37.6 years (and a standard deviation of 11.80 years. Before the beginning of arthrocentesis treatment, $85.7 \%$ of the patients $(n=12)$ reported severe pain, and $14.3 \%$ of the patients $(n=2)$ reported moderate pain; during the procedure, $57.1 \%(n=8)$ reported mild pain, and $42.9 \%(n=6)$ reported moderate pain; 1 year after arthrocentesis, 57.1\% $(n=8)$ of patients evolved into mild pain, and $35.7 \%(n=5)$ into moderate pain, and only 1 patient (7.1\%) still had severe pain (Table). When the frequency of pain intensity before and after the procedure was compared, a statistically significant difference $(p<0.0001)$ (Table) was observed. Means and standard deviations for pain intensity before and after the procedure also showed statistically significant differences ( $p$ < 0.0001) (Figure 1). On the improvement of mouth opening after arthrocentesis, $42.9 \%$ of patients $(\mathrm{n}=6)$ agreed that there was an improvement, $35.7 \%(n=5)$ completely agreed, $7.1 \%(n=1)$ disagreed, $7.1 \%$ totally disagreed, and $7.1 \%(n=1)$ expressed no opinion (Figure 2). Regarding the question on improvement of mastication after arthrocentesis, $42.9 \%$ of patients $(n=6)$ totally agreed, $35.7 \%(n=5)$ agreed, $7.1 \%(n=1)$ disagreed, $7.1 \%(n=1)$ totally disagreed, and $7.1 \%$ gave no opinion (Figure 3 ).

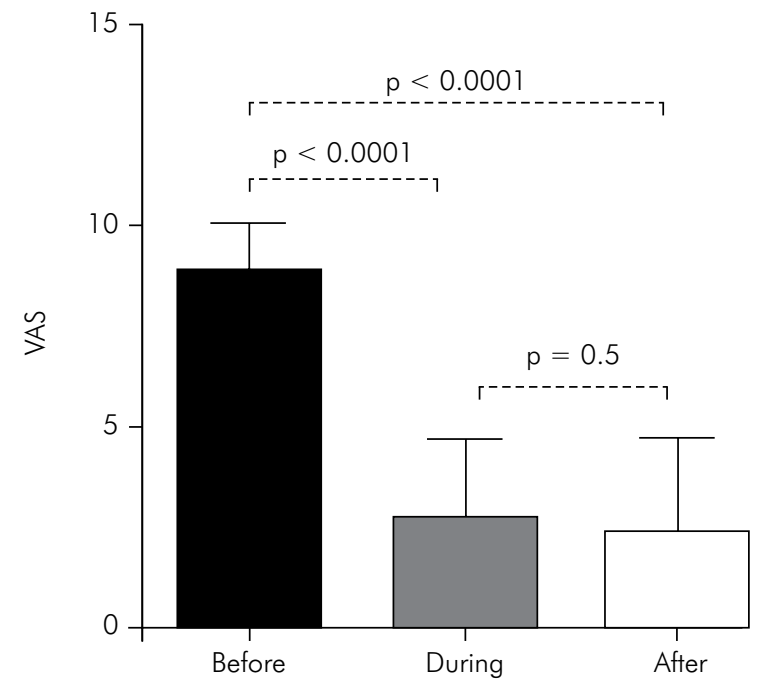

Figure 1. Mean \pm SD of VAS values before, during, and after treatment (Student $t$ test).

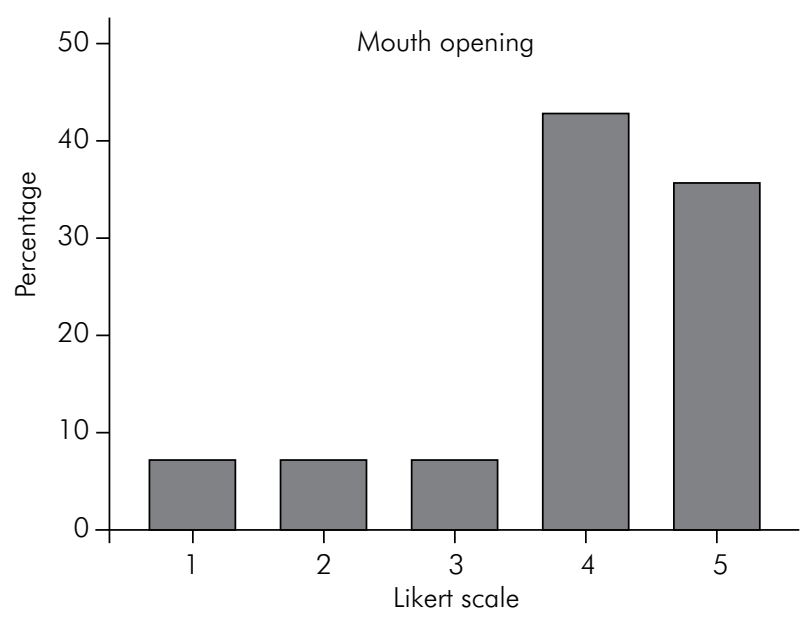

Figure 2. Frequency of values in the Likert scale as to the opening.

Table 1. (A) Distribution of pain intensity before, during and after arthrocentesis treatment (B) p-values for comparisons between groups.

\begin{tabular}{lccc}
\hline A & Before & During & After \\
\hline Mild & 0 & $8(57.1 \%)$ & $8(57.1 \%)$ \\
Moderate & $2(14.3 \%)$ & $6(42.9 \%)$ & $5(35.7 \%)$ \\
Severe & $12(85.7 \%)$ & 0 & $1(7.2 \%)$ \\
\hline B & Before vs During & Before vs After \\
\hline Mild + Moderate vs Severe & $<0.0001$ & $p^{*}$ & $<0.0001$ \\
Mild vs Moderate + Severe & 0.001 & 1.0 & 0.001 \\
\hline
\end{tabular}

* Chi-square test 


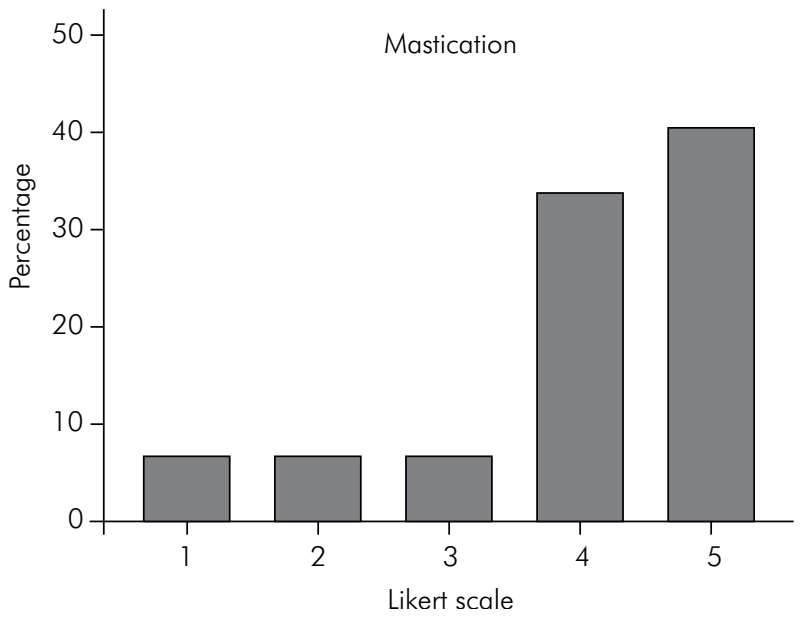

Figure 3. Frequency of values in the Likert scale as to mastication.

The linear regression graph showed a significant correlation between mouth opening and mastication values on the Likert scale $(\mathrm{R} 2=0.925, \mathrm{p}<0.0001)$ (Figure 4). Hundred percent of patients reported being satisfied with the treatment outcome.

\section{Discussion}

More recent literature supports the arthrocentesis procedure as the initial treatment of TMJ arthralgia in order to reduce pain and the functional deficit more

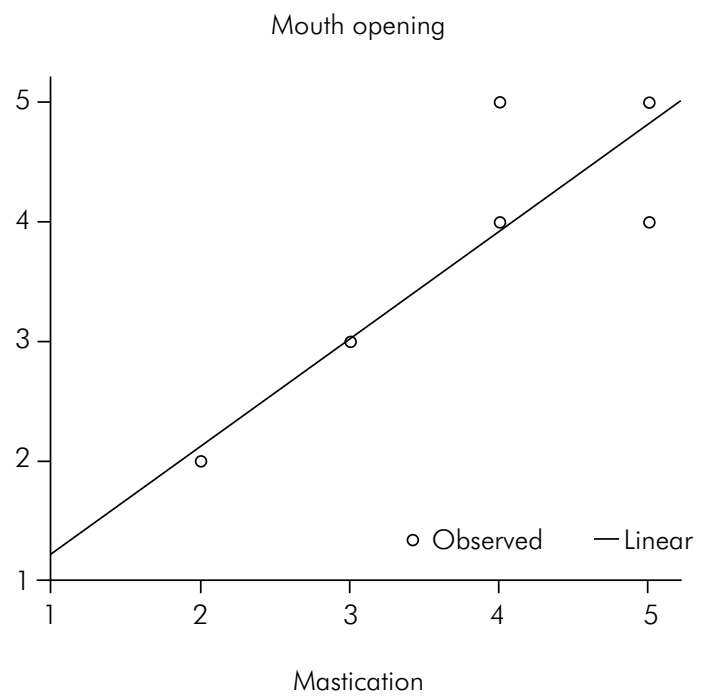

Figure 4. Correlation between Likert scale values associated to mouth opening and mastication (linear regression graph). quickly as compared to conventional therapy (occlusal splints, physiotherapy and anti-inflammatory drugs). Although conventional treatment can be as effective as arthrocentesis, the results will appear as late as 26 weeks after initiation. ${ }^{6}$ Therefore, both treatment modalities appear to be equally effective in the long term. ${ }^{6,78,9}$ In a literature review, Vos et al. ${ }^{9}$ claim that TMJ washing can be a valuable alternative in cases where the primary symptom is exacerbated pain. The present study chose to perform arthrocentesis because patients had a history of unsuccessful conventional therapies. Given the degree of anxiety and stress of these patients, $85.7 \%$ had severe pain, marked limitation of mouth opening and masticatory deficit, the therapy chosen was meant to address the complaints of patients in the short run, allowing an improvement of their seriously compromised quality of life.

Today, it is undeniable that both conventional therapy and arthrocentesis play an important role in TMJ arthralgia treatment, provided there is a systematic indication of each technique. For patients with acute pain and functional deficits, the authors recommend an initial conservative therapy, which includes the use of occlusal splints, anti-inflammatory medications, soft diet, and physiotherapy; however, if the signs and symptoms persist or if patients have underwent other treatments, arthrocentesis is recommended.

Patients with TMD show significantly altered emotional states, which are associated with a poorer quality of life (QOL). TMD is a major cause of chronic facial pain and is closely related to physical and psychosomatic stress, such as fatigue, sleep disturbances, anxiety and depression. The clinical picture of pain associated with TMD has been related to changes in QOL. ${ }^{10}$

All patients included in the study reported decreased QOL before treatment due to severe pain, difficulty chewing, decreased mouth opening and difficulty swallowing and speaking. After arthrocentesis, a significant improvement of QOL and clinically in these patients was observed. The survey of patient satisfaction ratified the treatment success, which was demonstrated by the total satisfaction of patients after 24 months.

\section{Conclusion}

The results of this study demonstrate that TMJ arthrocentesis is a viable treatment option for arthralgia 
relief in the short term. A reduction in symptom intensity gives the quality of life back to patients,

\section{References}

1. Craane B, Dijkstra PU, Stappaerts K, De Laat A. Randomized controlled trial on physical therapy for TMJ closed lock. J Dent Res. 2012 Apr;91(4):364-9

2. Su N, Yang X, Liu Y, Huang Y, Shi Z. Evaluation of arthrocentesis with hyaluronic acid injection plus oral glucosamine hydrochloride for temporomandibular joint osteoarthritis in oral-health-related quality of life. J Craniomaxillofac Surg. 2014 Sep;42(6):846-51. Epub 2013 Dec 25.

3. Brennan PA, Ilankovan V. Arthrocentesis for temporomandibular joint pain dysfunction syndrome. J Oral Maxillofac Surg. 2006 Jun;64(6):949-51.

4. Nitzan DW, Dolwick MF, Martinez GA. Temporomandibular joint arthrocentesis: a simplified treatment for severe, limited mouth opening. J Oral Maxillofac Surg. 1991 Nov;49(11):1163-7.

5. Alpaslan C, Dolwick MF, Heft MW. Five year retrospective evaluation of temporomandibular joint arthrocentesis. Int J Oral Maxillofac Surg. 2003 Jun;32(3):263-7.

6. Vos LM, Huddleston Slater JJ, Stegenga B. Arthrocentesis as initial treatment for temporomandibular joint arthropathy: which could be confirmed by the patients' total satisfaction with the procedures performed.

a randomized controlled trial. J Craniomaxillofac Surg. 2014 Jul;42(5):e134-9.

7. Yucel MA, Gozneli R, Alkumru HN, Kulak-Ozkan Y. Evaluating the additional effects of arthrocentesis on the condylar pathways of temporomandibular joint in patients with internal derangement treated with stabilizing splint. J Craniomaxillofac Surg. 2014 Jul;42(5):e86-90.

8. Diraçoğlu D, Saral IB, Keklik B, Kurt H, Emekli U, Ozçakar L, et al. Arthrocentesis versus nonsurgical methods in the treatment of temporomandibular disc displacement without reduction. Oral Surg Oral Med Oral Pathol Oral Radiol Endod. 2009 Jul;108(1):3-8.

9. Vos LM, Huddleston Slater JJ, Stegenga B. Lavage therapy versus nonsurgical therapy for the treatment of arthralgia of the temporomandibular joint: a systematic review of randomized controlled trials. J Orofac Pain. 2013 Spring;27(2):171-9.

10. Biasotto-Gonzalez DA, Mendes PCC, Jesus LA, Martins MD. Health-related quality of life in patients with temporomandibular disorder-a cross sectional study. Rev Inst Cienc Saude. 2009;27(2):128-32. Portuguese. 